\title{
Pattern Discovery Text Mining for Document Classification
}

\author{
Mustafa M. Shaikh \\ IT Department \\ Dr.D.Y.Patil Institute of \\ Engineering and Technology, \\ Ambi, Pune
}

\author{
Ashwini A. Pawar \\ IT Department \\ Dr.D.Y.Patil Institute of \\ Engineering and Technology, \\ Ambi, Pune
}

\author{
Vibha B. Lahane \\ Asst. Professor, IT Department \\ Dr.D.Y.Patil Institute of \\ Engineering and Technology, \\ Ambi, Pune
}

\begin{abstract}
In text documents data mining techniques have been discovered for mining useful patterns. But there are some questions, how to properly use and update discovered patterns is still an open research issue, specifically in the text mining. Therefore most existing text mining methods have used term-based approaches but they all suffer from the problems of polysemy (multiple meaning word) and synonymy(same meaning word).This is the literature survey paper with proposed system develops innovative and successful patternbased technique which contains the processes of pattern taxonomy, pattern deploying and gradually developing pattern ,to improve the effectiveness of using and updating researched patterns for finding applicable and interesting data with effectual patterns as per the users requirements. In this paper user is also getting the meaningful information without wrong meaning problem.
\end{abstract}

\section{General Terms}

Text mining, information filtering, Pattern Mining, Data Mining, pattern evolving, Text Classification.

\section{Keywords}

D-matrix ,Pattern Taxonomy

\section{INTRODUCTION}

Text mining is the discovery of interesting information in word documents. It is a stimulating issue to find precise information in text documents to help users to find what they want. Many applications, such as market analysis and business managing, can profit by the use of the data and facts extracted from a large amount of data. Knowledge discovery can be effectively use and update discovered patterns and apply it to field of text mining .Data mining is therefore an essential step in the process of knowledge discovery in databases, which means data mining is having all methods of knowledge discovery process and presenting modeling phase that is application of methods and algorithm for calculation of search pattern or models. These techniques include association rule mining(ASM), frequent item set mining(FIM), sequential pattern mining(SPM), maximum pattern mining(MPM) and closed pattern mining(CPM). Utmost of them are projected for the determination of developing efficient mining algorithms to find particular patterns within a equitable and tolerable time structure. With a great number of patterns engendered by using the data mining methods, how to effectively exploit these patterns is still an exposed research issue.

\section{BASIC CONCEPTS}

Taxonomy of entities for search engines is designed to improve significance ${ }^{[17]}$ in perpendicular search. Taxonomies of objects are trees whose nodes are labeled with entities which expect to occur in a web exploring request. These trees are used to compare keywords from search query with the keywords from answers (or snippets).Taxonomies, thesauri and concept hierarchies are crucial components for many applications of Natural Language processing, Information Retrieval and information management. Though, construction, regulation and handling taxonomies and ontologies is rather costly since a lot of manual processes are essential. A number of studies projected the programmed construction of taxonomies based on verbal resources and or statistical machine learning Web mining is one of the methods to form search engine taxonomies for net search. The taxonomy building procedure starts from the kernel objects and mines accessible source areas for new objects linked with these seed objects. New objects are molded by put on the machine learning to the present net search results for standing objects to form harmonies among them. These unity words then form parameters of present objects, and are revolved into new objects at the next learning repetition.

\section{SYSTEM ARCHITECTURE}
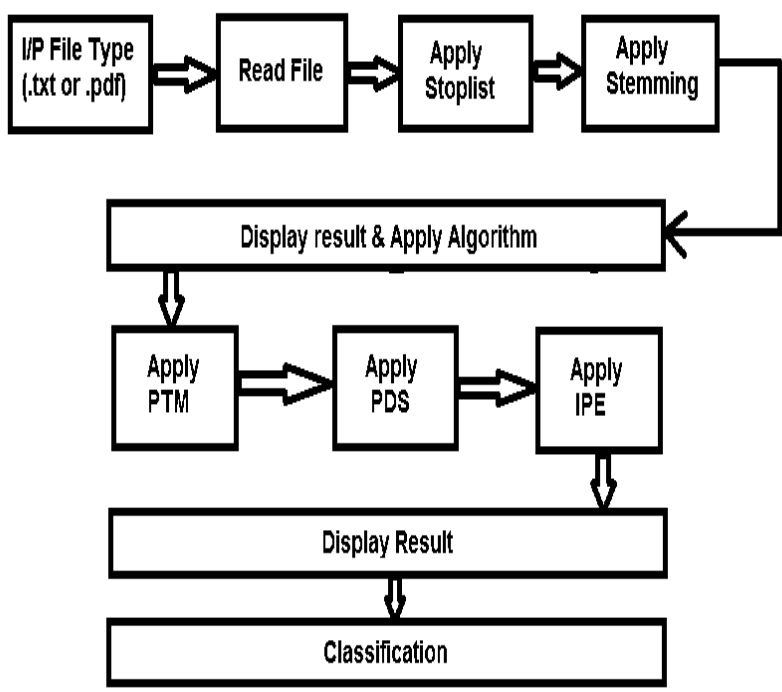

\section{PROPOSED WORK}

Research project selection is an important task for government and private research funding organizations. When a great number of research applications are received, it is mutual to cluster them conferring to their resemblances in research domains. The clustered proposals are then allotted to the suitable specialists for peer analysis. Present procedures for clustering proposals are built on manual toning of related research domain areas and/or keywords. ${ }^{[13]}$ However, the exact research domain areas of the offers cannot often be exactly nominated by the candidates due to their particular views and possible misapprehensions. So, rich info in the 
applications' full text can be used efficiently. Text-mining approaches have been projected to solve the problem by automatically categorizing text documents, largely in English.

\subsection{Preprocessing}

All words passes to pre-processing step. Inappropriate terms are removed there. This procedure is also called as tokenization procedure. It contains two types of processes such as stop list elimination, stem word elimination.

A). Stop List Elimination: Stop words are words which are cleaned out proceeding to, or afterward, treating of natural language data. They typically comprise prepositions, articles, and so on. There is no specific list of stop words for all applications and these stop words are controlled by the human but not automated. It saves the system assets. Stop word has list of arguments. That are considered as inappropriate and then it is eliminated .It consists of (a, an, the) articles, (for, in, at, etc.) preposition, etc.

B). Stem word removal: Stemming is the process for reducing inflected (or sometimes derived) words to their stem base or root form. It generally a written word forms. In this preprocess the text documents have to be processed using the Porter stemmer. It removes the Suffix's of the words these words are useful in the text mining for clustering the text documents in the text mining process users collects the documents and each documents are composed into the set of terms or words the words having stem have a same meaning in stem process the suffixes of the words, singular and plural words are considered into a one single word for meaning full text clustering process.

\subsection{Pattern Taxonomy Model}

Users assume that all documents are split into paragraphs. So a given document $A$ yields a set of paragraphs PS(A). Let B be a teaching set of docs, which contains a set of docs, B; Let $\mathrm{C}=\left\{\mathrm{c}_{1}, \mathrm{c}_{2}, \ldots, \mathrm{c}_{\mathrm{n}}\right\}$ be a set of terms (or keywords) which can be extracted from the set of documents, $\mathrm{B}$

T1:A set of paragraphs

\begin{tabular}{|c|c|}
\hline Paragraphs & Terms \\
\hline $\mathrm{Ap}_{1}$ & $\mathrm{c}_{1} \mathrm{c}_{2}$ \\
\hline $\mathrm{Ap}_{2}$ & $\mathrm{c}_{3} \mathrm{c}_{4} \mathrm{c}_{6}$ \\
\hline $\mathrm{Ap}_{3}$ & $\mathrm{c}_{3} \mathrm{c}_{4} \mathrm{c}_{5} \mathrm{c}_{6}$ \\
\hline $\mathrm{Ap}_{4}$ & $\mathrm{c}_{3} \mathrm{c}_{4} \mathrm{c}_{5} \mathrm{c}_{6}$ \\
\hline $\mathrm{Ap}_{5}$ & $\mathrm{c}_{1} \mathrm{c}_{2} \mathrm{c}_{6} \mathrm{c}_{7}$ \\
\hline $\mathrm{Ap}_{6}$ & $\mathrm{c}_{1} \mathrm{c}_{2} \mathrm{c}_{6} \mathrm{c}_{7}$ \\
\hline
\end{tabular}

A).Frequent and Closed Patterns-

Given ${ }^{[1]}$ a term set $D$ in document $d, \Gamma_{D} 7$ is used to denote the covering set of $\mathrm{D}$ for $\mathrm{A}$, which includes all paragraphs Ap PS(A) such that $\mathrm{D} \subseteq \mathrm{Ap}$, i.e.,

$$
\lceil D\rceil=\{\operatorname{Ap} \mid \operatorname{Ap} P S(A), D A p\} .
$$

Its absolute support is the number of occurrences of D in PS(A), that is $\left.\sup _{a}(D)=\mid \Gamma_{D}\right\urcorner \mid$.Its relative support is the fraction of the paragraphs that have the pattern, that is

$$
\sup _{\mathrm{r}}(\mathrm{D})=\frac{|\ulcorner\mathrm{D}\urcorner|}{|P S(A)|}
$$

A termset $D$ is called frequent pattern if its $\sup _{\mathrm{r}}$ (or $\left.\sup _{\mathrm{a}}\right) \geq \min \_$sup, minimum support.

Table 1 lists a set of paragraphs for a given document $A$, where $\operatorname{PS}(\mathrm{A})=\left\{\mathrm{Ap}_{1}, \mathrm{Ap}_{2}, \ldots ., \mathrm{Ap}_{6}\right\}$ and duplicate terms were removed.

T2: Frequent patterns and covering sets

\begin{tabular}{|c|c|}
\hline Frequent patterns & Covering sets \\
\hline$\left\{\mathbf{c}_{3}, \mathbf{c}_{\mathbf{4}}, \mathbf{c}_{\mathbf{6}}\right\}$ & $\left\{\mathrm{Ap}_{2}, \mathrm{Ap}_{3}, \mathrm{Ap}_{4}\right\}$ \\
\hline$\left\{\mathrm{c}_{3}, \mathrm{c}_{4}\right\}$ & $\left\{\mathrm{Ap}_{2}, \mathrm{Ap}_{3}, \mathrm{Ap}_{4}\right\}$ \\
\hline$\left\{\mathrm{c}_{3}, \mathrm{c}_{6}\right\}$ & $\left\{\mathrm{Ap}_{2}, \mathrm{Ap}_{3}, \mathrm{Ap}_{4}\right\}$ \\
\hline$\left\{\mathrm{c}_{4}, \mathrm{c}_{6}\right\}$ & $\left\{\mathrm{Ap}_{2}, \mathrm{Ap}_{3}, \mathrm{Ap}_{4}\right\}$ \\
\hline$\left\{\mathrm{c}_{3}\right\}$ & $\left\{\mathrm{Ap}_{2}, \mathrm{Ap}_{3}, \mathrm{Ap}_{3}, \mathrm{Ap}_{4}\right\}$ \\
\hline$\left\{\mathrm{c}_{4}\right\}$ & $\left\{\mathrm{Ap}_{1}, \mathrm{Ap}_{5}, \mathrm{Ap}_{6}\right\}$ \\
\hline$\left\{\mathbf{c}_{\mathbf{1}}, \mathbf{c}_{\mathbf{2}}\right\}$ & $\left\{\mathrm{Ap}_{2}, \mathrm{Ap}_{3}, \mathrm{Ap}_{4}\right\}$ \\
\hline$\left\{\mathrm{c}_{1}\right\}$ & $\left\{\mathrm{Ap}_{2}, \mathrm{Ap}_{3}, \mathrm{Ap}_{4}\right\}$ \\
\hline$\left\{\mathrm{c}_{2}\right\}$ & $\left\{\mathrm{Ap}_{2}, \mathrm{Ap}_{3}, \mathrm{Ap}_{4}, \mathrm{Ap}_{5}, \mathrm{Ap}_{6}\right\}$ \\
\hline$\left\{\mathrm{c}_{6}\right\}$ &
\end{tabular}

Let min_sup $=50 \%$, users can obtain ten frequent patterns in Table 1 using the above explanations. T2 illuminates the ten frequent patterns and their covering sets. Not all repeated patterns in $\mathrm{T} 2$ are beneficial. For example, pattern $\left\{\mathrm{c}_{3}, \mathrm{c}_{4}\right\}$ always occurs with term $c_{6}$ in paragraphs, i.e., the shorter pattern, $\left\{c_{3}, c_{4}\right\}$, is always a part of the larger pattern, $\left\{c_{3}, c_{4}\right.$, $\left.c_{6}\right\}$, in all of the paragraphs. Therefore, users consider that the smaller one, $\left\{c_{3}, c_{4}\right\}$, is a noise pattern and expect to keep the larger pattern, $\left\{\mathrm{c}_{3}, \mathrm{c}_{4}, \mathrm{c}_{6}\right\}$, only. Given a term set $\mathrm{D}$, its covering set $\left.\mid \Gamma_{D}\right\urcorner \mid$ is a subset of paragraphs. Similarly, given a set of paragraphs $\mathrm{E} \subseteq$ PS (A), users can define its term set, which satisfies

termset $(\mathrm{E})=\{c \mid \forall A p \in E \rightarrow c \in A p\}$

The closure of is defined as follows:

$$
\left.\mathrm{Cls}(\mathrm{D})=\operatorname{termset}\left({ }^{\mathrm{C}} \mathrm{D}\right\urcorner\right) \text {. }
$$

A pattern $\mathrm{D}$ (also a termset) is called closed if and only if $\mathrm{D}=\mathrm{Cls}(\mathrm{D})$.

Let $\mathrm{D}$ be a closed pattern. Users can prove that

$$
\sup _{a}(\mathrm{D} 1)<\sup _{\mathrm{a}}(\mathrm{D})
$$

for all patterns D1 $\supset$ D; otherwise, if $\sup _{a}(D 1)=\sup _{a}(D)$, users have

$$
\lceil D 1\rceil=\lceil D\rceil
$$

Where $\sup _{a}(\mathrm{D} 1)$ and $\sup _{\mathrm{a}}(\mathrm{D})$ are the absolute support of pattern D1 and D, respectively.

\section{Users also have}

$\operatorname{Cls}(\mathrm{D})=\operatorname{termset}(\lceil D\rceil)=\operatorname{termset}(\lceil D 1\rceil) \supseteq \mathrm{D} 1 \supset \mathrm{D}$,

that is, $\mathrm{Cls}(\mathrm{D}) \neq \mathrm{D}$. 


\section{B). Pattern Taxonomy-}

Patterns can be structured into a taxonomy by using the is-a (or subset) relative. For the example of T1, where users have demonstrated a set of paragraphs of a document, and the exposed 10 repeated patterns in T2 if assuming min_sup = $50 \%$. There are, however, only threeclosed patterns in this example. They are $\left\langle\mathrm{c}_{3}, \mathrm{c}_{4}, \mathrm{c}_{6}\right\rangle,\left\langle\mathrm{c}_{1}, \mathrm{c}_{2}\right\rangle$, and $\left\langle\mathrm{c}_{6}\right\rangle$.

Fig. 1 illustrates an example of the pattern taxonomy for the frequent patterns in $\mathrm{T} 2$, where the nodes denote repeated patterns and their covering sets; non-closed patterns can be pruned; the edges are "is-a" relation. After pruning, some direct "is-a" retaliations may be changed, for example, pattern $\left\{\mathrm{c}_{6}\right\}$ would become a direct sub-pattern of $\left\{\mathrm{c}_{3}, \mathrm{c}_{4}, \mathrm{c}_{6}\right\}$, after pruning non-closed patterns. Smaller patterns in the taxonomy, for example pattern $\left\{\mathrm{c}_{6}\right\}$ (see Fig. 1) are usually more general because they could be used frequently in both positive and documents; and larger patterns, for example pattern $\left\{\mathrm{c}_{3}, \mathrm{c}_{4}, \mathrm{c}_{6}\right\}$ in the taxonomy are usually more specific since they may be used only in confident documents. The semantic info will be used in the pattern taxonomy to develop the performance of using closed patterns in text mining.

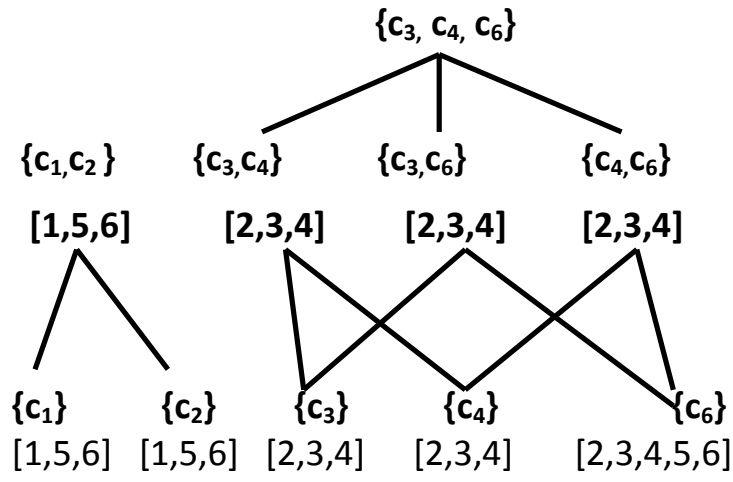

Fig 1: Pattern taxonomy

\section{C). Closed Sequential Patterns}

$\mathrm{A}^{[2]}$ sequential pattern $\mathrm{s}=\left\langle\mathrm{c}_{1}, \ldots, \mathrm{c}_{\mathrm{r}}\right\rangle(\mathrm{ci} \in \mathrm{C})$ is an ordered list of terms. A sequence $\mathrm{s}_{1}=\left\langle\mathrm{d}_{1}, \ldots, \mathrm{d}_{\mathrm{i}}\right\rangle \mathrm{is}$ a subsequence of another sequence $s_{2}=\left\langle e_{1}, \ldots, e_{j}\right\rangle$, denoted by $s_{1} \hat{O} \quad s_{2}$, if $\exists j_{1}$, . $\ldots, \mathrm{j}_{\mathrm{y}}$ such that $1 \leq \mathrm{j}_{1}<\mathrm{j}_{2} \ldots<\mathrm{j}_{\mathrm{e}} \leq \mathrm{j}$ and $\mathrm{d}_{1}=\mathrm{e}_{\mathrm{j} 1}, \mathrm{~d}_{2}=\mathrm{e}_{\mathrm{j} 2}, \ldots$, $\mathrm{d}_{\mathrm{i}}=\mathrm{e}_{\mathrm{je}}$. Given s1 Ô $\mathrm{s} 2$, users usually say $\mathrm{s} 1$ is a sub pattern of $s_{2}$, and $s_{2}$ is a super pattern of $s_{1}$. In the following, users simply say patterns for sequential patterns. Given a pattern (an ordered termset) $\mathrm{D}$ in document $\mathrm{A},\lceil D\rceil$

is still used to denote the covering set of $\mathrm{D}$, which includes all

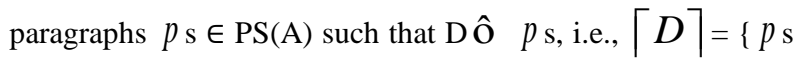
$\mid p \mathrm{~s} \in \mathrm{PS}(\mathrm{A}), \mathrm{D} \hat{\mathrm{O}} p \mathrm{~s}\}$.Its absolute support is the number of occurrences of $\mathrm{D}$ in $\mathrm{PS}(\mathrm{A})$, that is $\sup _{\mathrm{a}}(\mathrm{D})=|\lceil D\rceil|$. Its relative ${ }^{[3]}$ support is the fraction of the paragraphs that contain the pattern, that is,

$$
\sup _{\mathrm{r}}(\mathrm{D})=\frac{\mid\left\lceil\mathrm{D}_{\urcorner} \mid\right.}{|P S(A)|} .
$$

A sequential pattern $\mathrm{D}$ is called frequent pattern if its relative support (or absolute support) _ min sup, a minimum support. The property of closed patterns (see eq. (1)) can be used to define closed sequential patterns. A frequent sequential pattern $\mathrm{D}$ is called closed if not 9 any superpatternD1 of $\mathrm{D}$ such that $\sup _{a}(D 1)=\sup _{a}(D)$.

\subsection{Pattern Deploying Method}

In demand to use the semantic info in the pattern taxonomy to develop the performance of closed patterns in text mining, users need to interpret discovered patterns by summarizing them as d-patterns (see the definition below) in order to accurately evaluate term weights (supports).

The rationale behind this motivation is that d-patterns include more semantic meaning than terms that are selected based on a term-based technique (e.g., tf*idf). As a result, a term with a higher $\mathrm{tf} *$ idf value could be meaningless if it has not cited by some d-patterns (some important parts in documents). The evaluation of term weights (supports) is different to the normal term-based methods. In the term-based methods, the estimate of term weights is based on the distribution of terms in documents. In this ,terms are biased conferring to their forms in exposed closed patterns.

\section{A). Representation of Closed Patterns-}

It is complicated to derive a method to apply discovered patterns in text documents for information purifying systems. To make simpler this procedure, users first analyze the composition operation $\oplus$ defined in [25]. Let $p_{1}$ and $p_{2}$ be sets of term-digit pairs. $\mathrm{p} 1 \oplus \mathrm{p} 2$ is called the composition of $\mathrm{p} 1$ and p2 which fulfills

$$
\begin{aligned}
& \mathrm{p}_{1} \oplus \mathrm{p}_{2}=\left\{\left(\mathrm{c}, \mathrm{d}_{1}+\mathrm{d}_{2}\right) \mid\left(\mathrm{c}, \mathrm{d}_{1}\right) \in \mathrm{p}_{1},\left(\mathrm{c}, \mathrm{d}_{2}\right) \in \mathrm{p}_{2}\right\} \\
& \cup\left\{(\mathrm{c}, \mathrm{d}) \mid(\mathrm{c}, \mathrm{d}) \in \mathrm{p}_{1} \cup \mathrm{p}_{2}, \operatorname{not}\left(\left(\mathrm{c},{ }_{-}\right) \in \mathrm{p}_{1} \cap \mathrm{p}_{2}\right)\right\}
\end{aligned}
$$

where is the wild card that matches any number. For the special case users have $p_{1} \oplus=p$; and the operands of the composition operation are exchangeable. The result of the composition is quiet a set of term-number pairs.

For example,

$$
\left\{\left(c_{1}, 1\right),\left(c_{2}, 2\right),\left(c_{3}, 3\right)\right\} \oplus\left\{\left(c_{2}, 4\right)\right\}=\left\{\left(c_{1}, 1\right),\left(c_{2}, 6\right),\left(c_{3}, 3\right)\right\}
$$

Or

$\left\{\left(\mathrm{c}_{1}, 2 \%\right),\left(\mathrm{c}_{2}, 5 \%\right),\left(\mathrm{c}_{3}, 9 \%\right)\right\} \oplus\left\{\left(\mathrm{c}_{1}, 1 \%\right),\left(\mathrm{c}_{2}, 3 \%\right)\right\}=\left\{\left(\mathrm{c}_{1}, 3 \%\right),\left(\mathrm{c}_{2}, 8\right.\right.$ $\left.\%),\left(c_{3}, 9 \%\right)\right\}$

Formally, for all positive documents $\mathrm{A}_{\mathrm{i}} \in \mathrm{D}^{+}$, users first deploy its closed patterns on a common set of terms $\mathrm{T}$ in order to obtain the following d-patterns (deployed patterns, nonsequential weighted patterns):

$$
\hat{A}_{\mathrm{i}}=\left\{\left(\mathrm{c}_{\mathrm{i} 1}, \mathrm{n}_{\mathrm{i} 1}\right),\left(\mathrm{c}_{\mathrm{i} 2}, \mathrm{n}_{\mathrm{i} 2}\right), \ldots \ldots,\left(\mathrm{c}_{\mathrm{im}}, \mathrm{n}_{\mathrm{im}}\right)\right\}
$$

Where $c_{i j}$ in pair $\left(c_{i j}, n_{i j}\right)$ denotes a single term and $n_{i j}$ is its support in $A_{i}$ which is the total absolute supports given by closed patterns that contain $\mathrm{c}_{\mathrm{ij}}$; or $\mathrm{n}_{\mathrm{ij}}$ (simply in this paper) is the total number of closed patterns that contain $\mathrm{c}_{\mathrm{ij}}$.

For example, using Fig. 1 and T1, users have

$$
\begin{aligned}
& \sup _{\mathrm{a}}\left(\left\langle\mathrm{c}_{3}, \mathrm{c}_{4}, \mathrm{c}_{6}\right\rangle\right)=3, \\
& \sup _{\mathrm{a}}\left(\left\langle\mathrm{c}_{1}, \mathrm{c}_{2}\right\rangle\right)=3, \\
& \sup _{\mathrm{a}}\left(\left\langle\mathrm{c}_{6}\right\rangle\right)=5 \text {, and }
\end{aligned}
$$

$\hat{A}=\left\{\left(\mathrm{c}_{1}, 3\right),\left(\mathrm{c}_{2}, 3\right),\left(\mathrm{c}_{3}, 3\right),\left(\mathrm{c}_{6}, 8\right)\right\}$

The process of calculating d-patterns can be easily described by using the $\oplus$ operation in Algorithm 1 (PTM) displayed in Fig. 2, where a term's support is the total number of closed patterns that contain the term. Users also can obtain the dpatterns of the five sample documents in which are expressed as follows: 
$\hat{A}_{1}=\{($ carbon, 2$),($ emiss, 1$),($ air, 1$),($ pollut, 1$)\}$,

$\hat{A}_{2}=\{($ greenhouse, 1$),($ global, 2$),($ emiss, 1$)\}$,

$\hat{A}_{3}=\{($ greenhouse, 1$),($ global,$=1),($ emiss, 1$)\}$,

$\hat{A}_{4}=\{($ carbon, 1$),(\operatorname{airl}, 2),(\operatorname{antarct}, 1)\}$,

$\hat{A}_{5}=\{($ emiss, 1$),($ global, 1$),($ pollut, 1$)\}$.

Let AAP be a set of d-patterns in $\mathrm{D}^{+}$, and $\mathrm{p} \in \mathrm{AAP}$ be a dpattern. Users call $\mathrm{p}(\mathrm{c})$ the absolute support of term $\mathrm{c}$, which is the number of patterns that contain $\mathrm{c}$ in the corresponding patterns taxonomies. In order to efficiently install patterns in dissimilar taxonomies from the different positive documents, d-patterns will be normalized using the following assignment sentence:

$$
p(c) \longleftarrow p(c) \times \frac{1}{\sum_{c \in C} p(c)}
$$

Actually the relationship between d-patterns and terms can be explicitly described as the following association mapping [25], a set-value function:

$\beta: \mathrm{AAP} \rightarrow 2^{\mathrm{C} \times[0,1]}$

such that

$\beta\left(p_{\mathrm{i}}\right)=\left\{\left(\mathrm{c}_{1}, \omega_{1}\right),\left(\mathrm{c}_{2}, \omega_{2}\right), \ldots \ldots,\left(\mathrm{c}_{\mathrm{k}}, \boldsymbol{\omega}_{\mathrm{k}}\right)\right\}$

for all $p_{\mathrm{I}} \in \mathrm{AAP}$, where

$$
p_{\mathrm{i}}=\left\{\left(\mathrm{c}_{1}, \mathrm{f}_{1}\right),\left(\mathrm{c}_{2}, \mathrm{f}_{2}\right), \ldots \ldots,\left(\mathrm{c}_{\mathrm{k}}, \mathrm{f}_{\mathrm{k}}\right)\right\} \in \mathrm{AAP}, \omega_{i}=\frac{f_{i}}{\sum_{j=1}^{k} f_{j}}
$$

\section{And $\mathrm{C}=\{\mathrm{c} \mid(\mathrm{c}, \mathrm{f}) \in \mathrm{p}, \mathrm{p} \in \mathrm{AAP}\}$}

$\beta\left(p_{i}\right)$ is called the normal form (or normalized d-pattern)

of d-pattern $p_{\mathrm{I}}$ in this paper, and

termset $\left(p_{\mathrm{i}}\right)=\left\{\mathrm{c}_{1}, \mathrm{c}_{2}, \ldots, \mathrm{c}_{\mathrm{k}}\right\}$

\subsection{Inner Pattern Evaluation}

In this section, users ${ }^{[4]}$ talk over how to restructuring supports of terms within normal forms of d-patterns centered on documents in the training set. The method will be useful to lessen the side effects of noisy patterns for the reason that of the low-frequency problem. This method is called inner pattern evolution here, for the reason that it only changes a pattern's term supports within the pattern.

A threshold is usually used to categorize documents into appropriate or inappropriate groups. Using the d-patterns, the threshold can be well-defined naturally as follows:

Threshold(AAP) $=\min _{p \in A A P}\left(\sum_{(c, \omega) \in \beta(p)} \operatorname{support}(\mathrm{c})\right)$

A noise negative document $\mathrm{A}$ in $\mathrm{D}$ is a document that the system misleadingly recognized as a positive, that is weight (A) $\geq$ Threshold(AAP). In order to lessen the noise, users must track which d-patterns have been used to provide rise to such a mistake. Users call these patterns offenders of

nd.An offender of $\mathrm{A}$ is a d-pattern that has minimum one term in A. The set of offenders of A is defined by:

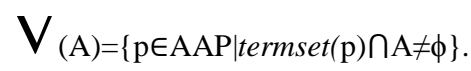

There are two sorts of offenders: 1) a complete conflict offender which is a subset of $\mathrm{A}$; and 2) a partial conflict offender which holds part of terms of A.The basic idea of bring up-to-date patterns is described as follows:

Complete conflict offenders are detached from d-patterns first. For partial conflict offenders, their term supports are restructured in order to lessen the effects of noise documents. The main procedure of inner pattern evolution is executed by the algorithm IPEvolving. The input of this method is se of patterns .The output is a serene of d-pattern. Step 2 in IPEvolving is used to guess the threshold for discovery of the noise documents. Steps 3to 10 reread term supports by using all noise documents. Step 4 is to find noise documents and the equivalent offenders. Step 5 gets normal forms of dpatterns NDP. Step 6 calls algorithm scuffling to update NDP agreeing to noise documents. Steps 7 to 9 compose updated normal forms organized. The time complexity of Algorithm 2 is defined by step 2, the number of calls for Scuffling algorithm and the number of using $\oplus$ operation. Step 2 takes (nm).

For each noise pattern A, the algorithm catches its offenders that takes $\mathrm{O}(\mathrm{nm} \times|\mathrm{nd}|)$ in step 4 , and then calls once Scuffling. After that, it calls $\mathrm{n} \oplus$ operation that takes

$$
\mathrm{O}(\mathrm{nmm})=\mathrm{O}(\mathrm{nm})^{2} \text {. }
$$

The task of algorithm Scuffling is to adjust the support supply of terms within a d-pattern. A different strategy is committed in this algorithm for each type of offender. As stated in step 2 in the algorithm Scuffling, complete conflict offenders (dpatterns) are detached since all elements within the d-patterns are held by the documents representing that they can be thrown away for preventing interfering from these possible "noises." The parameter proposing is used in step 4 for the purpose of provisionally keeping the cheap supports of some terms in a partial conflict offender. The offering is part of the sum of supports of terms in a d-pattern where these terms also act in a noise document. The algorithm calculates the base in step 5 which is definitely not zero since termset $(\mathrm{p})-\mathrm{d} \neq \phi$; and then updates the support allocations of terms in step 6.

For example, for the following d-pattern

$$
\hat{A}=\left\{\left(\mathrm{c}_{1}, 3\right),\left(\mathrm{c}_{2}, 3\right),\left(\mathrm{c}_{3}, 3\right),\left(\mathrm{c}_{3}, 4\right),\left(\mathrm{c}_{6}, 8\right)\right\} \text {. }
$$

Its normal form is

$$
\left\{\left(c_{1}, 3 / 20\right),\left(c_{2}, 3 / 20\right),\left(c_{3}, 3 / 20\right),\left(c_{4}, 3 / 20\right),\left(c_{6}, 2 / 5\right)\right\}
$$

Assume $\mathrm{nd}=\left\{\mathrm{c}_{1}, \mathrm{c}_{2}, \mathrm{c}_{6}, \mathrm{c}_{9}\right\}, \hat{A}$ will be a partial conflict offender since

termset $(\hat{A}) \cap \mathrm{nd}==\{\mathrm{t} 1, \mathrm{t} 2, \mathrm{t} 6\} \neq \phi$

Let $\mu=2$,

offering $=\frac{1}{2} \times\left(\frac{3}{20}+\frac{3}{20}+\frac{2}{5}\right)=\frac{7}{20}$, and

base $=\frac{3}{20}+\frac{3}{20}=\frac{7}{10}$ Hence, users can get the

Following restructured normal form by using algorithm $\left\{\left(c_{1}, 3 / 40\right),\left(c_{2}, 3 / 40\right),\left(c_{3}, 13 / 40\right),\left(c_{4}, 13 / 40\right),\left(c_{6}, 1 / 5\right)\right\}$
Scuffling: 
Let $m=|T|, n=|D|$ the number of positive documents in a training set, and $\mathrm{q}$ be the number of noise documents in D. The time complexity of algorithm Scuffling is decided by steps 6 to 9. For a given noise document A, its time complexity is $\mathrm{O}\left(\mathrm{nm}^{2}\right)$ if let $\mathrm{A}=\mathrm{A} \cap \mathrm{T}$, where $\mathrm{T}=$ $\{t \in$ termset( $p) \mid p \in D P\}$. Therefore, the time complexity of algorithm Scuffling is $\mathrm{O}\left(\mathrm{nm}^{2}\right)$ for a given noise document. Based on the above study, the total time complexity of the inner pattern evolution is $\mathrm{O}\left(n m+q\left(n m|n d|+n m^{2)=} \mathrm{O}\left(q n m^{2}\right)\right.\right.$ bearing in mind that the noise document A can be replaced by $A \cap T$ before leading the pattern evolution. The projected model contains two phases: the training phase and the testing phase. In the training phase, the suggested model first calls Algorithm PTM (D, min sup) to find d-patterns in documents (D) based on a min sup, and assesses term supports by deploying d-patterns to terms. It also calls Algorithm IPEvolving (D, DP, $\mu$ ) to reread term supports using noise documents in $\mathrm{D}$ based on an trial coefficient $\mu$. In the testing phase, it assesses weights for all entering documents using eq. (4). The entering documents then can be organized based on these weights.

\section{ALGORITHMS USED}

\section{${ }^{[11]}$ Algorithm 1: SPMining(PL,min_sup)}

Input: a list of nTerms frequent sequential pattern $P L$ : minimum support min_sup.

Output: a set of sequential patterns $S P$.

\section{Method:}

1: $S P \leftarrow S P \leftarrow\left\{P_{a} \in \mid \exists P_{b} \in P L\right.$ such a that len $\left(P_{a}\right)=\operatorname{len}\left(\mathrm{P}_{\mathrm{b}}\right)-1$

$\left.\left.\wedge P_{a} \mathrm{c} \mathrm{P}_{\mathrm{b}} \wedge \operatorname{supp}_{\mathrm{a}}\left(P_{a}\right)=\operatorname{supp}_{\mathrm{a}}\left(P_{b}\right)\right\}\right\} \|$ pattern mining

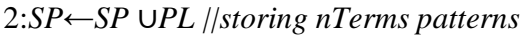

3: $P L^{\prime} \leftarrow \varnothing$

4: for each pattern $p$ in $P L$ do begin

5: generating p-projected database $P D$

6: for each frequent term $t$ in $P D$ do begin

7: $P^{\prime}=\mathrm{p} \bowtie \mathrm{t}||$ sequence extension

8: if $\operatorname{supp}_{\mathrm{r}}\left(P^{\prime}\right) \geq$ min_sup then

9: $\quad P L^{\prime} \leftarrow P L^{\prime} \cup P^{\prime}$

10: $\quad$ end if

11: end for

12: end for

13: if $\left|P L^{\prime}\right|=0$ then

14: return ||no more pattern

15: else

16: $\quad$ call SPMining $\left(P L^{\prime}\right.$, min_sup $)$

17: end if

18: output $S P$

Algorithm 2: $\mathrm{PDM}\left(D, \min \_s u p\right)$

Input: a list of document $D$ : minimum support min_sup.

Output: a set of vectors $\Delta$

\section{METHOD:}

1: $\Delta \leftarrow \emptyset$

2: for each document $d$ in $D$ do begin

3: extract $l$ Terms frequent patterns $P L$ from $d$

4: $S P=\operatorname{SPMining}(P L$, min_sup $) \quad \|$ Call Algorithm 1

5: $\stackrel{\text { ut }}{d} \leftarrow \varnothing$

6: for each pattern $p$ in $S P$ do begin

7: $\stackrel{\text { ü }}{d} \leftarrow \stackrel{\text { ü }}{d} \oplus P^{\prime} \| P^{\prime}$ is the expanded form of $p$

8: end for

9: $\Delta \leftarrow \Delta \cup\{\stackrel{m}{d}\}$

10: end for

Algorithm 3: $\operatorname{PDS}(S P)$

Input: a set of frequent sequential patterns SP.

Output: a sevectors of feature in expanded form $d$.

METHOD:
1: sum_supp $=0, \stackrel{\text { ut }}{d} \leftarrow \varnothing$
2: for each pattern $p$ in $S P$ do begin
3: $s u m \_s u p p+=\sup _{a}(p)$
4: end for
5: for each pattern $p$ in $S P$ do begin
6: $f=\operatorname{supp}_{a}(p) /\left(\operatorname{sum} \_s u p p \times \operatorname{len}(p)\right)$
7: $P^{\prime} \leftarrow \varnothing$
8: for each term $t$ in $p$ do begin
9: $P^{\prime} \leftarrow P^{\prime} \cup\{(t, f)\}$
10: end for
11: $\stackrel{\text { ü }}{d} \leftarrow \stackrel{\text { u }}{d} \oplus P^{\prime}$
12: end for

Algorithm 4: DPEvolving $(\Omega, D)$

Input: a list of deployed patterns $\Omega$; a list of documents $D$

Output: a set of term weight pairs $\stackrel{\text { ü }}{d}$.

METHOD:

1: $\stackrel{\text { ut }}{d} \leftarrow \phi / /$ estimate minimum threshold

2: $\tau=$ Threshold $(D)$

3: for each document $d$ in $D$ do begin

4: if Threshold $(\{d\})>\tau$ then

5: $\Delta_{\mathrm{p}}=\{$ Ap $\in \Omega \mid$ termset $(\mathrm{Ap}) \cap \mathrm{d} \neq \varnothing\}$

6: Shuffling $\left(\mathrm{d}, \Delta_{\mathrm{p}}\right)$

7: end if 
8: for each deployed pattern $\mathrm{d}$ in $\Omega$ do begin

9: $\stackrel{\text { ü }}{d} \leftarrow \stackrel{\text { d }}{\mathrm{d}} \oplus A p$

10: end for

11: end for

Algorithm 5: Shuffling (d, $\left.\Delta_{\mathrm{p}}\right)$

Input: a document $\mathrm{d}$ and a list of deployed patterns $\Delta_{\mathrm{p}}$

Output: updated deployed patterns

\section{METHOD:}

1: for each deployed pattern $d$ in $\Delta_{\mathrm{p}}$ do begin

2: if termset $(d) \subseteq \mathrm{d}$ then // complete conflict offender

3: $\Omega=\Omega-\{$ Ap $\}$

4: else // partial conflict offender

5:offering' $=\left(1-\frac{1}{\mu}\right) \times \sum_{t \in \text { termset }(A p)}\{t$ weight $\mid t \in d\}$

6: base $=\sum_{t \in \text { termset }(A p)}\{$ t.weight $\mid t \notin d\}$

7: for each term $t$ in termset $(A p)$ do begin

8: if $t \in d$ then // shrink offender weight

9: $t$.weight $=\frac{1}{\mu} \times t$.weight

10: else //shuffle weights

11: $t$. weight $=t$. weight $\times\left(1+\right.$ offering ${ }^{\prime} \div$ base $)$

12: end if

13: end for

14: end if

15: end for

\section{EXPERIMENTAL RESULTS}

T3: The List of Methods Used for Evaluation

\begin{tabular}{|l|l|c|}
\hline Method & Description & Algorithm \\
\hline Sequential ptns & $\begin{array}{l}\text { Data mining method } \\
\text { using sequential } \\
\text { patterns }\end{array}$ & SPM \\
\hline $\begin{array}{l}\text { Sequential closed } \\
\text { ptns }\end{array}$ & $\begin{array}{l}\text { Data mining method } \\
\text { using freq. sequential } \\
\text { closed patterns }\end{array}$ & SCPM \\
\hline Freq. Itemset & $\begin{array}{l}\text { Data mining method } \\
\text { using freq.itemset }\end{array}$ & NSPM \\
\hline Freq.closed itemset & $\begin{array}{l}\text { Data mining method } \\
\text { using freq.closed } \\
\text { itemset }\end{array}$ \\
\hline
\end{tabular}

\section{ACKNOWLEDGMENTS}

The authors wish to thank Prof. Vibha Lahane from Dr. D Y Patil Institute of engineering and Technology, Ambi, Pune. Authors also wish to thank Ning Zhong, Yuefeng Li, and Sheng-Tang $\mathrm{Wu}$ for providing such a deep research on this topic. 


\section{REFERENCES}

[1] Manan Parikh1, Bharat Chaudhari2 and Chetna Chand 2013 "A Comparative Study of Sequential Pattern Mining Algorithms"

[2] Jian Pei, Member, IEEE Computer Society, Jiawei Han, Senior Member, IEEE, BehzadMortazavi-Asl, Jianyong Wang, Helen Pinto, Qiming Chen, UmeshwarDayal, Member, IEEE Computer Society, and Mei-Chun Hsu 2004 "Mining sequential Patterns by Pattern-Growth: The Prefix Span Approach"

[3] Jian Pei, Jiawei Han, and Runying Mao 2010 "CLOSET: An Efficient Algorithm for Mining Often Closed Item sets"

[4] RamshankarChoudhary Prof. AkhtarRasool Dr. NilayKhare2012 "Variation of Boyer-Moore String Matching Algorithm: A Comparative Analysis"

[5] li-ping jing,hou-kuan huang,hong-bo shi 2004 "improved feature selection approach tfidf intext mining"

[6] Robert Burbidge, Bernard Buxton2000's An Introduction to Support Vector Machines for DataMining

[7] Z. Yang,W. H. Tang,A. Shintemirov, and Q. H. Wu 2009 "Association Rule Mining-Based Dissolved Gas Analysis for Fault Diagnosis of Power Transformers"
[8] Gerard Salton and Christopher Buckley 1988 "Termweighting Approaches in Automatic Text Retrival"

[9] Tsang-Hsiang Cheng and Chih-Ping Wei 2008 "A Clustering-Based Approach for Integrating DocumentCategory Hierarchies"

[10] RupaliBhaisare,T. RajuRao 2013 "Review On Text Mining With Pattern Discovery".

[11] NingZhong, Yuefeng Li, and Sheng-Tang Wu 2012 "Effective Pattern Discovery for Text Mining"

[12] JoydipDatta 2010 "Ranking in Information Retrieval"

[13] KjerastiAas Line Eikvil 1999 "Text categorization: a Survey"

[14] RamkrishnanShrikant, RakeshAgrawal "Mining Generelizedassotiation"

[15] Chih-Ping Wei and Yu-Hsiu Chang March 2007 "Discovering Event Evaluation Patterns From Document Sequences"

[16] Laura AuriaRouslanA.Moro August 2008 "Support Vector Machines (SVM) as a Technique for Solvency Analysis"

[17] http://en.wikipedia.org/wiki/Taxonomy_for_search_engi nes 